\title{
Intravoxel water diffusion heterogeneity imaging of human high-grade gliomas
}

\author{
Thomas C. Kwee a, Craig J. Galbán ${ }^{b}$, Christina Tsien', Larry Junckd, \\ Pia C. Sundgren ${ }^{a}$, Marko K. Ivancevic ${ }^{a, e}$, Timothy D. Johnson ${ }^{f}$, \\ Charles R. Meyer ${ }^{b}$, Alnawaz Rehemtulla ${ }^{b}$, Brian D. Ross ${ }^{b}$ \\ and Thomas L. Chenevert ${ }^{\mathrm{a} *}$
}

This study aimed to determine the potential value of intravoxel water diffusion heterogeneity imaging for brain tumor characterization and evaluation of high-grade gliomas, by comparing an established heterogeneity index ( $\alpha$ value) measured in human high-grade gliomas to those of normal appearing white and grey matter landmarks. Twenty patients with high-grade gliomas prospectively underwent diffusion-weighted magnetic resonance imaging using multiple b-values. The stretched-exponential model was used to generate $\alpha$ and distributed diffusion coefficient (DDC) maps. The $\alpha$ values and DDCs of the tumor and contralateral anatomic landmarks were measured in each patient. Differences between $\alpha$ values of tumors and landmark tissues were assessed using paired $t$-tests. Correlation between tumor $\alpha$ and tumor DDC was assessed using Pearson's correlation coefficient. Mean $\alpha$ of tumors was significantly lower than that of contralateral frontal white matter $(p=0.0249)$, basal ganglia $(p<0.0001)$, cortical grey matter $(p<0.0001)$, and centrum semiovale $(p=0.0497)$. Correlation between tumor $\alpha$ and tumor DDC was strongly negative (Pearson correlation coefficient, $-0.8493 ; p<0.0001$ ). The heterogeneity index $\alpha$ of human high-grade gliomas is significantly different from those of normal brain structures, which potentially offers a new method for evaluating brain tumors. The observed negative correlation between tumor $\alpha$ and tumor DDC requires further investigation. Copyright (c) 2009 John Wiley \& Sons, Ltd.

Keywords: diffusion-weighted magnetic resonance imaging; stretched-exponential; intravoxel water diffusion heterogeneity; brain tumor; glioma

\section{INTRODUCTION}

The incidence of gliomas is around five to ten per 100000 in the general population $(1,2)$. High-grade [World Health Organization (WHO) grade III and IV] astrocytomas are the most common gliomas, with glioblastomas being about four times more common than anaplastic astrocytomas $(1,2)$. Despite decades of intensive clinical and laboratory research, high-grade gliomas are still considered incurable, with a poor median survival (1 year in glioblastomas and 2-3 years in anaplastic astrocytomas) $(1,2)$.

* Correspondence to: T. L. Chenevert, Department of Radiology, University of Michigan Medical Center, B2A209 UH 1500 East Medical Center Drive, Ann Arbor, MI 48109, USA.

E-mail: tlchenev@umich.edu

a T. C. Kwee, P. C. Sundgren, M. K. Ivancevic, T. L. Chenevert Department of Radiology, University of Michigan Medical Center, Ann Arbor, $M I, U S A$

b C. J. Galbán, C. R. Meyer, A. Rehemtulla, B. D. Ross, A. Rehemtulla, B. D. Ross Center for Molecular Imaging, University of Michigan Medical Center, Ann Arbor, MI, USA

c C. Tsien

Department of Radiation Oncology, University of Michigan Medical Center, Ann Arbor, MI, USA

d L. Junck

Department of Neurology, University of Michigan Medical Center, Ann Arbor, MI, USA
Since currently available imaging techniques are not sufficiently specific, final diagnosis and tumor grade still have to be established by histopathological examination of tumor biopsy samples or by surgical resection $(1,2)$. More accurate preoperative grading of primary brain tumors could increase the diagnostic yield of brain biopsies, thereby improving patient management. Another contributing factor to the poor survival of patients with high-grade gliomas is the inability of currently available imaging techniques to accurately delineate the tumor, as a result of which targeted focal treatments may not be effective (3). In addition,

e M. K. Ivancevic

Philips Healthcare, MR Clinical Science, Cleveland, $\mathrm{OH}$, USA

f T. D. Johnson

Department of Biostatistics, University of Michigan Medical Center, Ann Arbor, MI, USA

Contract/grant sponsor: National Institutes of Health and the National Cancer Institute; contract/grant numbers: P01CA85878; P01CA59827; 1P01CA87634; R24CA83099; P50CA93990.

Abbreviations used: $D D C$, distributed diffusion coefficient; $D W I$, diffusionweighted magnetic resonance imaging; EPI, echo-planar imaging; FDG-PET, 18F-fluoro-2-deoxyglucose positron emission tomography; FLAIR, fluidattenuated inversion recovery; $M P G$, motion probing gradient; $M R$, magnetic resonance; $P R M_{A D G}$ parametric response mapping of diffusion; $R O I$, region of interest; SD, standard deviation; SE, spin-echo; SENSE, SENSitivity Encoding; SNR, signal-to-noise ratio; SPIR, spectral presaturation inversion recovery; SS, single-shot; VOI, volume of interest; WHO, World Health Organization. 
conventional imaging is not able to give an early assessment of the effectiveness of radiation and/or chemotherapy (3). Another unsolved issue is the distinction between radiation-induced necrosis from tumor recurrence (3). Early identification of patients with a poor treatment response or who suffer from tumor recurrence can be of great advantage: it provides the opportunity to adjust individual treatment regimes more rapidly, and sparing patients unnecessary morbidity, expense and delay in initiation of effective treatment. The application of diffusion-weighted magnetic resonance (MR) imaging (DWI) for brain tumor characterization and evaluation of gliomas is under active investigation (3-5). Evidence regarding the effectiveness of DWI regarding brain tumor characterization, tumor delineation, and differentiation between radiation-induced necrosis and tumor recurrence is either conflicting or scarce (3), while a voxel-based quantitative DWI approach [functional diffusion mapping, currently referred to as parametric response mapping of diffusion $\left.\left(P R M_{A D C}\right)\right]$ has recently shown promise of giving an early prediction of survival in patients with high-grade gliomas $(4,5)$.

Diffusion in biological tissue is most frequently quantified using a mono-exponential model. However, diffusion-weighted signal decay in the brain and in brain tumors has been shown to be multi-exponential (6), and the bi-exponential model may be a better way to describe the admixture of multiple exponential signal decays (6). However, the bi-exponential model is probably also an oversimplification of reality, and it is more realistic to assume a larger number $(>2)$ of intravoxel proton pools with different diffusion coefficients (7). Moreover, detailed studies of animal and human brain tissue have determined that assumptions of fast and slow compartments being extra- and intracellular water pools, respectively, are not supported by empirical data (8).

To overcome the difficulty of making assumptions about the number of intravoxel proton pools with different diffusion coefficients in biological tissue, Bennett et al. $(7,9,10)$ introduced the stretched-exponential model. The stretched-exponential model is described as follows:

$$
S(b) / S_{o}=\exp \left(-(b \times D D C)^{\alpha}\right),
$$

where $S(b)$ is the signal magnitude with diffusion weighting $b, S_{0}$ is the signal magnitude with no diffusion weighting, $b$ is the b-value, the index $\alpha$ relates to intravoxel water diffusion heterogeneity, varying between 0 and 1 , and the $D D C$ is the distributed diffusion coefficient, representing mean intravoxel diffusion rates. Interestingly, this model introduces a new parameter $(\alpha)$, which provides a new type of image contrast (different from conventional DWI), that relates to the degree of intravoxel water diffusion heterogeneity. By inspection of equation (1), it should be clear an $\alpha=1$ is equivalent to mono-exponential diffusion-weighted signal decay, thus low intravoxel diffusion heterogeneity. Conversely an $\alpha$ near 0 indicates a higher degree of multi-exponential signal decay $(7,9,10)$. This convention maintains consistency with Bennett and colleagues' $(7,9,10)$ definition of $\alpha$ as a heterogeneity index, although we remind the reader that a numerically high $\alpha$ index (i.e. $\alpha$ approaching 1) represents low intravoxel diffusion heterogeneity approaching mono-exponential decay, and a numerically low $\alpha$ index (i.e. $\alpha$ approaching 0 ) represents a high degree of diffusion heterogeneity exhibited as multi-exponential decay. Another key point worth emphasis is that the term 'heterogeneity' in this context refers to intravoxel heterogeneity of exponential decay, as opposed to intervoxel heterogeneity of diffusion coefficients as often is the case particularly in tumor. After fitting the stretched-exponential model to the acquired DWI data, pixel-by-pixel $\alpha$ and DDC maps can be obtained, allowing for visual and (regional) quantitative evaluation of $\alpha$ values and DDCs, respectively $(7,9,10)$.

So far, the stretched-exponential model has only been investigated in animal models and healthy volunteers $(7,9,10)$. Since high-grade gliomas are histologically more heterogeneous than normal brain tissue $(1,2,11)$, we hypothesized that $\alpha$ values in high-grade gliomas are lower than those in normal brain structures. The purpose of this study was therefore to determine the potential diagnostic value of intravoxel heterogeneity imaging for brain tumor characterization and evaluation of high-grade gliomas, by comparing $\alpha$ values of human high-grade gliomas to those of anatomic landmarks.

\section{MATERIALS AND METHODS}

\section{Patients}

This study was approved by the local institutional review board and written informed consent was obtained from all participants. Twenty patients with high-grade glioma [ten men, ten women, mean age 58.2 years (range, 20-89 years), WHO grade III: $n=3$, WHO grade IV: $n=17$, mean tumor volume $23.4 \mathrm{ml}$ (range 0.4-88.6 ml), temporal lobe as primary tumor location: $n=11$, frontal lobe as primary tumor location: $n=4$, occipital lobe as primary tumor location: $n=3$, parietal lobe as primary tumor location: $n=2$ ] prospectively underwent DWI of the brain, before any treatment was started. Exclusion criteria were general contraindications to MR imaging, such as implanted pacemaker and claustrophobia.

\section{MR imaging}

All patients were examined with a 3.0 T MR scanner (Achieva 3.0 T Quasar Dual, Philips Healthcare, Best, The Netherlands) using a eight-channel head coil. DWI was performed using a single-shot (SS) spin-echo (SE) echo-planar imaging (EPI) sequence, with the following parameters: repetition time/echo time of $8700 / 60 \mathrm{~ms}$, image acquisiton in the axial plane, slice thickness/gap of 4/ $1 \mathrm{~mm}$, number of slices of 28 , field of view of $240 \times 240 \mathrm{~mm}$, acquisition matrix of $128 \times 99$, motion probing gradients (MPGs) in three orthogonal axes, b-values of $0,1000,2000$, and $4000 \mathrm{~s} /$ $\mathrm{mm}^{2}$, number of signal averages of 1 (for b-value of $0 \mathrm{~s} / \mathrm{mm}^{2}$ ), 2 (for b-value of $1000 \mathrm{~s} / \mathrm{mm}^{2}$ ), and 3 (for b-values of 2000 and $4000 \mathrm{~s} / \mathrm{mm}^{2}$ ), phase encode reduction of $77.8 \%$, half scan factor of 0.733 , parallel imaging (SENSitivity Encoding [SENSE]) factor of 3, EPI factor of 35, spectral presaturation inversion recovery (SPIR) fat suppression, actual voxel size of $1.88 \times 2.41 \times 4.00 \mathrm{~mm}^{3}$, reconstructed voxel size of $0.94 \times 0.94 \times 4.00 \mathrm{~mm}^{3}$, and total scan time of $4 \mathrm{~min}$ and $30 \mathrm{~s}$. In all patients, routine anatomical pre- and post- post-gadolinium 3D T1-weighted fast field echo, turbo SE axial T2-weighted, and turbo SE axial fluid-attenuated inversion recovery (FLAIR) sequences were performed in addition to axial DWI sequences.

\section{Image analysis}

The stretched-exponential model [equation (1)] was fitted to the obtained DWI data using a nonlinear least-squares routine, to 
create whole-brain isotropic $\alpha$ and DDC maps $(7,9,10)$. Noise thresholds were set to restrict diffusion calculation to only pixels safely above background noise to avoid fitting voxels with low signal-to-noise ratio (SNR). Freehand volumes of interest (VOIs) were placed in the tumor and in four contralateral anatomic landmarks (frontal white matter, basal ganglia (putamen and head of caudate nucleus), cortical grey matter, and centrum semiovale) in each patient by one of the authors (T.C.K.). Tumor VOls were contoured on post-gadolinium T1-weighted images. If a cystic (resection) cavity was present, it was included within the tumor VOI if circumscribed by contrast enhancement and excluded if outside the enhancing region. Furthermore, all regions with an impeded diffusion relative to the surrounding brain parenchyma were included in the tumor VOls. All other MR sequences were used to identify (apparently) unaffected contralateral anatomic landmarks. Care was taken to avoid inclusion of parts of the ventricles and subarachnoid cisterns into the VOls, in order to minimize partial volume averaging effects between tumor/anatomic landmarks and the relatively fastdiffusing cerebrospinal fluid (7). When identifying tumor and anatomic landmarks and creating corresponding VOls, the observer was blinded to $\alpha$ and DDC maps. Subsequently, defined VOls were applied to $\alpha$ and DDC maps, and $\alpha$ values and DDCs of tumor and anatomic landmarks were automatically calculated. All image processing and analysis was performed on in-house software developed in Matlab (The Mathworks, Inc., Natick, MA).

\section{Statistical analysis}

Differences between $\alpha$ values of tumors and $\alpha$ values of contralateral anatomic landmarks, and differences between DDCs of tumors and DDCs of contralateral anatomic landmarks were assessed using paired $t$ tests. Due to findings by Bennett et al. (9), which showed in an animal model of glioma that intrasubject variability in the $\alpha$ value was higher in tumor and peritumor VOIs than in either grey or white matter, differences between standard deviations (SDs) of $\alpha$ values of tumors and contralateral anatomic landmarks were also assessed using paired $t$-tests. Correlation between tumor $\alpha$ and tumor DDC was assessed using Pearson's correlation coefficient. $P$-values $<0.05$ were considered statistically significant. Statistical analyses were executed using MedCalc Software (MedCalc, Mariakerke, Belgium).

\section{RESULTS}

All measurements could be performed, except for the frontal white matter contralateral to the tumor in one patient, which was affected by edema. Results are displayed in Table 1. Mean $\alpha$ of tumors was significantly lower than mean $\alpha$ of contralateral

Table 1. Mean \pm SD $\alpha$ values and DDCs (in $10^{-3} \mathrm{~mm}^{2} / \mathrm{s}$ ) of different brain structures and tumor

\begin{tabular}{|lcc|}
\hline Tissue & $\alpha$ & DDC \\
\hline Frontal white matter & $0.62 \pm 0.01$ & $0.80 \pm 0.09$ \\
Basal ganglia & $0.80 \pm 0.03$ & $0.64 \pm 0.05$ \\
Cortical grey matter & $0.72 \pm 0.03$ & $0.72 \pm 0.04$ \\
Centrum semiovale & $0.62 \pm 0.02$ & $0.70 \pm 0.05$ \\
Tumor & $0.58 \pm 0.08$ & $1.64 \pm 0.71$ \\
\hline
\end{tabular}

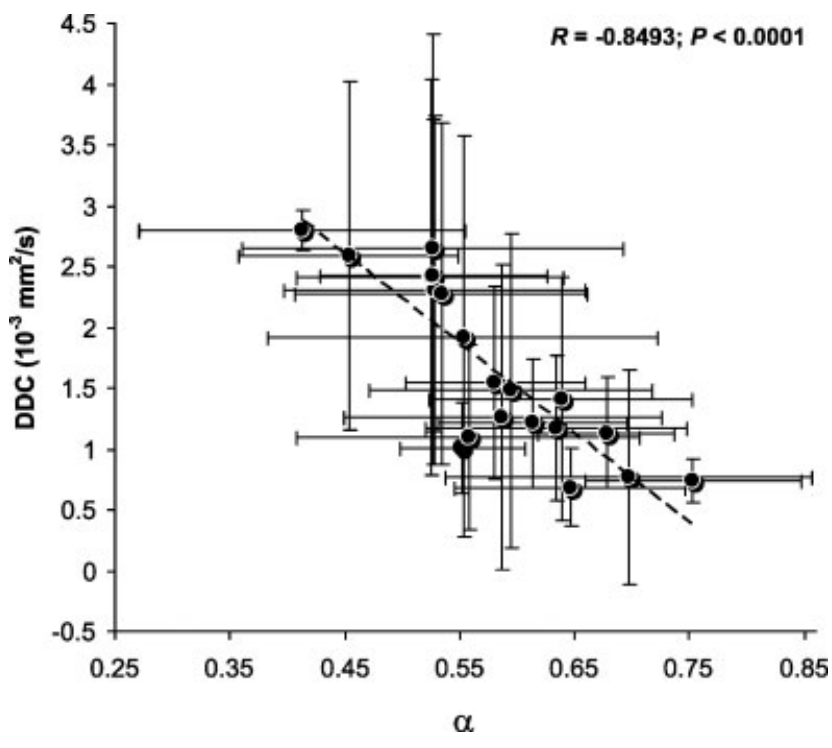

Figure 1. Scatter plot with mean tumor $\alpha$ (x-axis) vs. mean tumor DDC (y-axis) including the dashed regression line. Error bars represent $\pm S D$ of each tumor VOI.

frontal white matter $(p=0.0249)$, basal ganglia $(p<0.0001)$, cortical grey matter $(p<0.0001)$, and centrum semiovale $(p=0.0497)$. Mean DDC of tumors was significantly higher than mean DDC of contralateral frontal white matter $(p=0.0001)$, basal ganglia ( $p<0.0001)$, cortical grey matter $(p<0.0001)$, and centrum semiovale $(p<0.0001)$. Mean SD of $\alpha$ values of tumors was significantly higher than mean SD of $\alpha$ values of contralateral frontal white matter $(p<0.0001)$, basal ganglia $(p<0.0001)$, cortical grey matter $(p=0.0123)$, and centrum semiovale $(p<0.0001)$. The correlation between tumor $\alpha$ and tumor DDC, which was strongly negative (Pearson correlation coefficient, $-0.8493 ; P<0.0001)$ is presented in Figure 1. Figures $2-5$ present representative examples of $\alpha$ and DDC maps of high-grade gliomas. Examples of the stretched-exponential model fit to target tissues (white matter, grey matter, basal ganglia, and tumor) are illustrated in Figure 6.

\section{DISCUSSION}

In this study, we found that $\alpha$ values of human high-grade gliomas were significantly lower than those of (apparent) normal brain structures. This finding can be explained by the fact that high-grade gliomas, in particular glioblastoma multiforme $(85 \%$ of included patients), are associated with considerable histological heterogeneity $(1,2,11)$; the high degree of cellular pleomorphism (i.e. high variability in the size and shape of cells and cellular nuclei), the presence of intravoxel microscopic cystic/ necrotic foci, and intravoxel heterogeneity in vascular structures may lead to the existence of a high number of different compartments with different proton pools (i.e. lower $\alpha$ values) in high-grade gliomas, relative to normal, unaffected brain tissue. This hypothesis is further supported by the fact that SD of $\alpha$ values of high-grade gliomas was significantly higher than that of contralateral anatomic landmarks, which reflects their variable histological heterogeneity; similar results were reported in an animal model of glioma (9). Furthermore, in the present study 


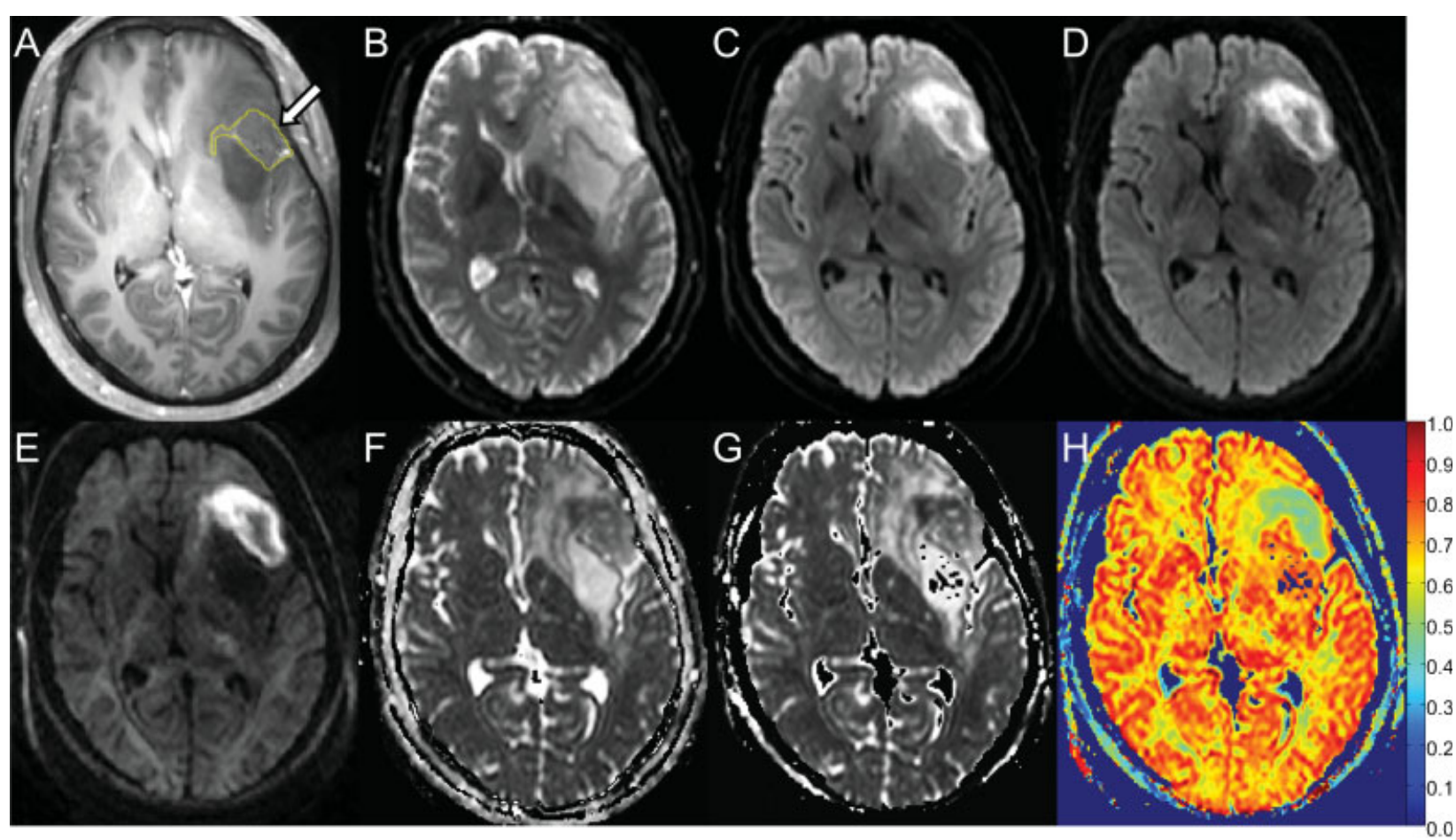

Figure 2. A 28-year-old male with a high-grade glioma (WHO grade III) in the left temporal lobe. Axial post-gadolinium T1-weighted image with tumor VOI (a), diffusion-weighted images at b-values of $0 \mathrm{~s} / \mathrm{mm}^{2}$ (b), $1000 \mathrm{~s} / \mathrm{mm}^{2}$ (c), $2000 \mathrm{~s} / \mathrm{mm}^{2}$ (d), and $4000 \mathrm{~s} / \mathrm{mm}^{2}$ (e), and isotropic ADC (f), DDC (g), and $\alpha$ (h) maps show the tumor (arrow). This tumor had a mean DDC of $1.01 \times 10^{-3} \mathrm{~mm}^{2} / \mathrm{s}$ and a mean $\alpha$ value of 0.55 . Note the good contrast between the tumor and the surrounding brain tissue on the $\alpha$ map $(\mathrm{H})$.
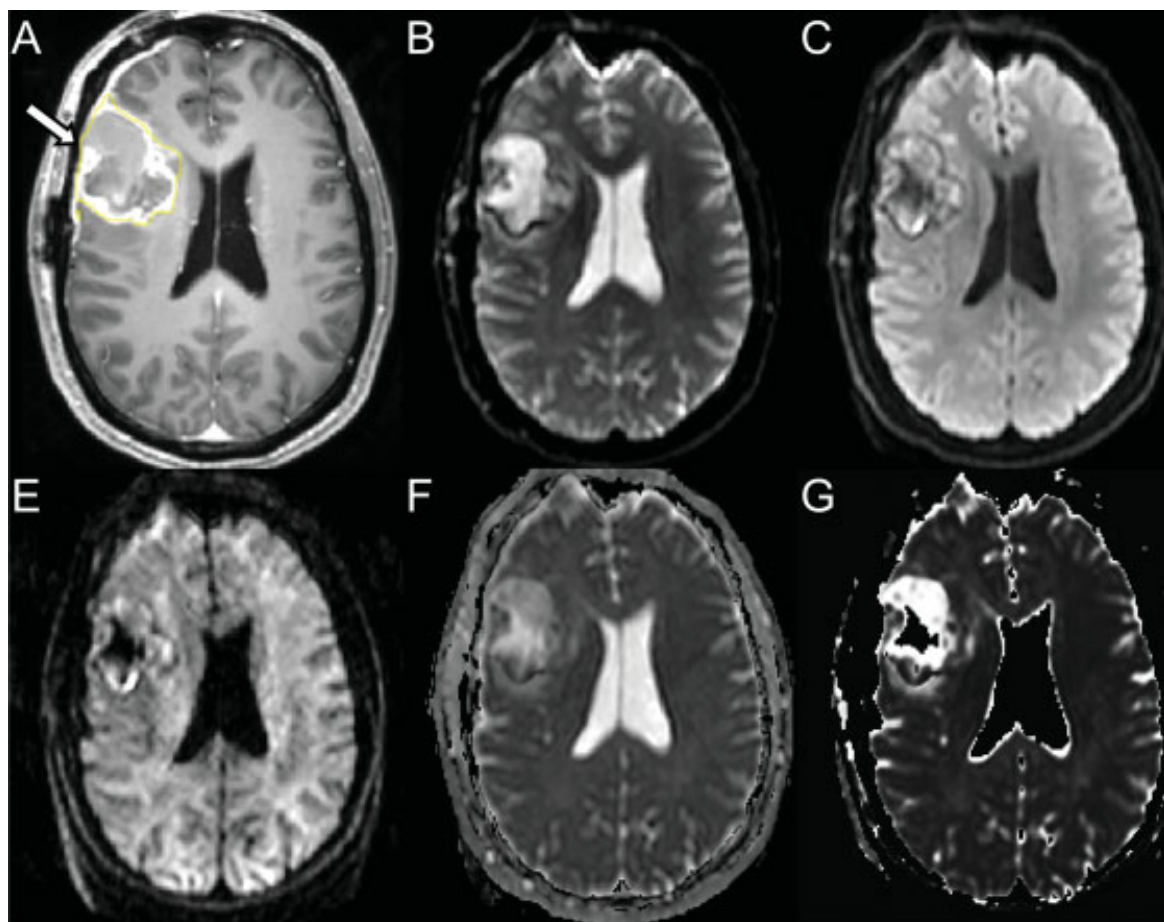

D

G
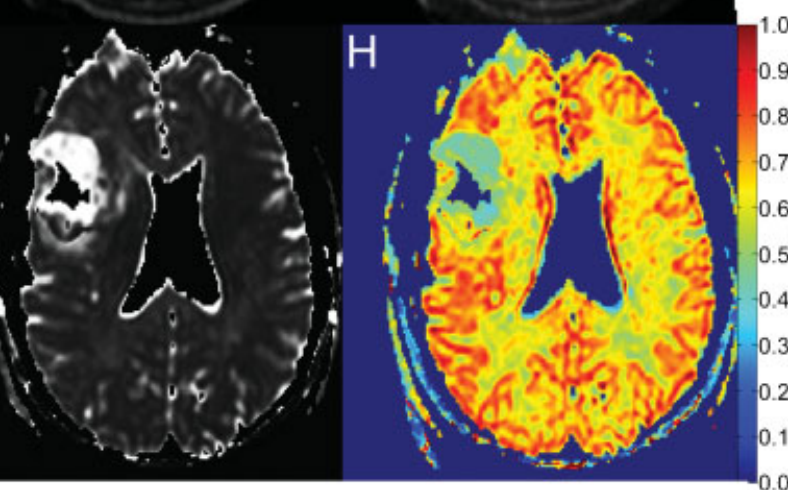

Figure 3. A 46-year-old male with a high-grade glioma (WHO grade IV) in the right frontal lobe. Axial post-gadolinium T1-weighted image with tumor VOI (a), diffusion-weighted images at b-values of $0 \mathrm{~s} / \mathrm{mm}^{2}$ (b), $1000 \mathrm{~s} / \mathrm{mm}^{2}$ (c), $2000 \mathrm{~s} / \mathrm{mm}^{2}$ (d), and $4000 \mathrm{~s} / \mathrm{mm}^{2}$ (e), and isotropic ADC (f), DDC (g), and $\alpha$ (h) maps show the tumor (arrow). This tumor had a mean DDC of $2.66 \times 10^{-3} \mathrm{~mm}^{2} / \mathrm{s}$ and a mean $\alpha$ value of 0.53 . Note the good contrast between the tumor and the surrounding brain tissue on the $\alpha$ map $(\mathrm{H})$. 


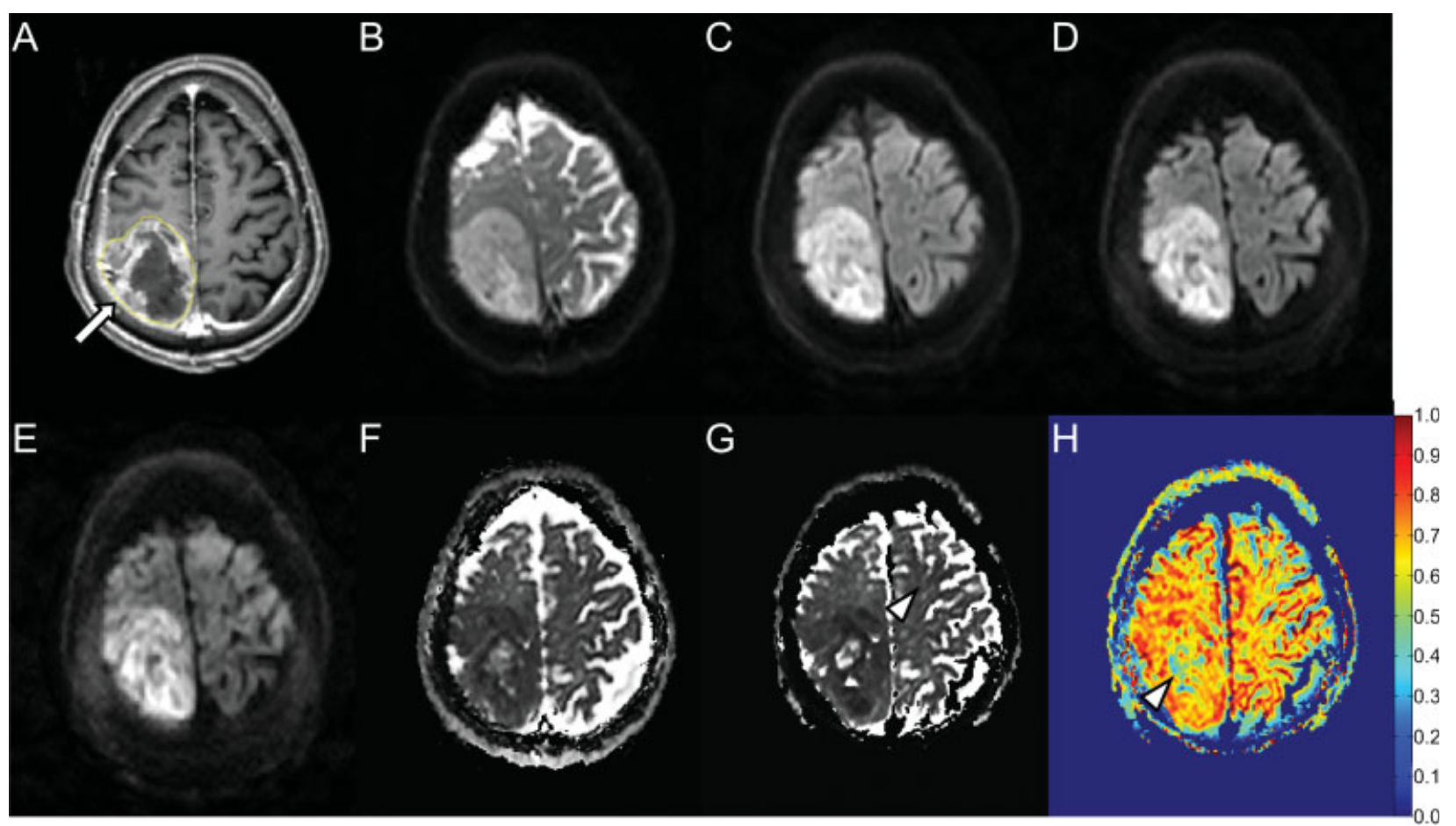

Figure 4. A 61-year-old male with a high-grade glioma (WHO grade IV) in the right parietal lobe. Axial post-gadolinium T1-weighted image with tumor VOI (a), diffusion-weighted images at b-values of $0 \mathrm{~s} / \mathrm{mm}^{2}$ (b), $1000 \mathrm{~s} / \mathrm{mm}^{2}$ (c), $2000 \mathrm{~s} / \mathrm{mm}^{2}$ (d), and $4000 \mathrm{~s} / \mathrm{mm}^{2}$ (e), and isotropic ADC (f), DDC (g), and $\alpha$ (h) maps show the tumor (arrow). This tumor had a mean DDC of $0.68 \times 10^{-3} \mathrm{~mm}^{2} / \mathrm{s}$ and a mean $\alpha$ value of 0.65 . In this case, overall contrast between the tumor and surrounding brain tissue was moderate on the $\alpha$ map $(\mathrm{H})$. Nevertheless, the center of the tumor clearly exhibited a lower $\alpha$ value (arrowhead).
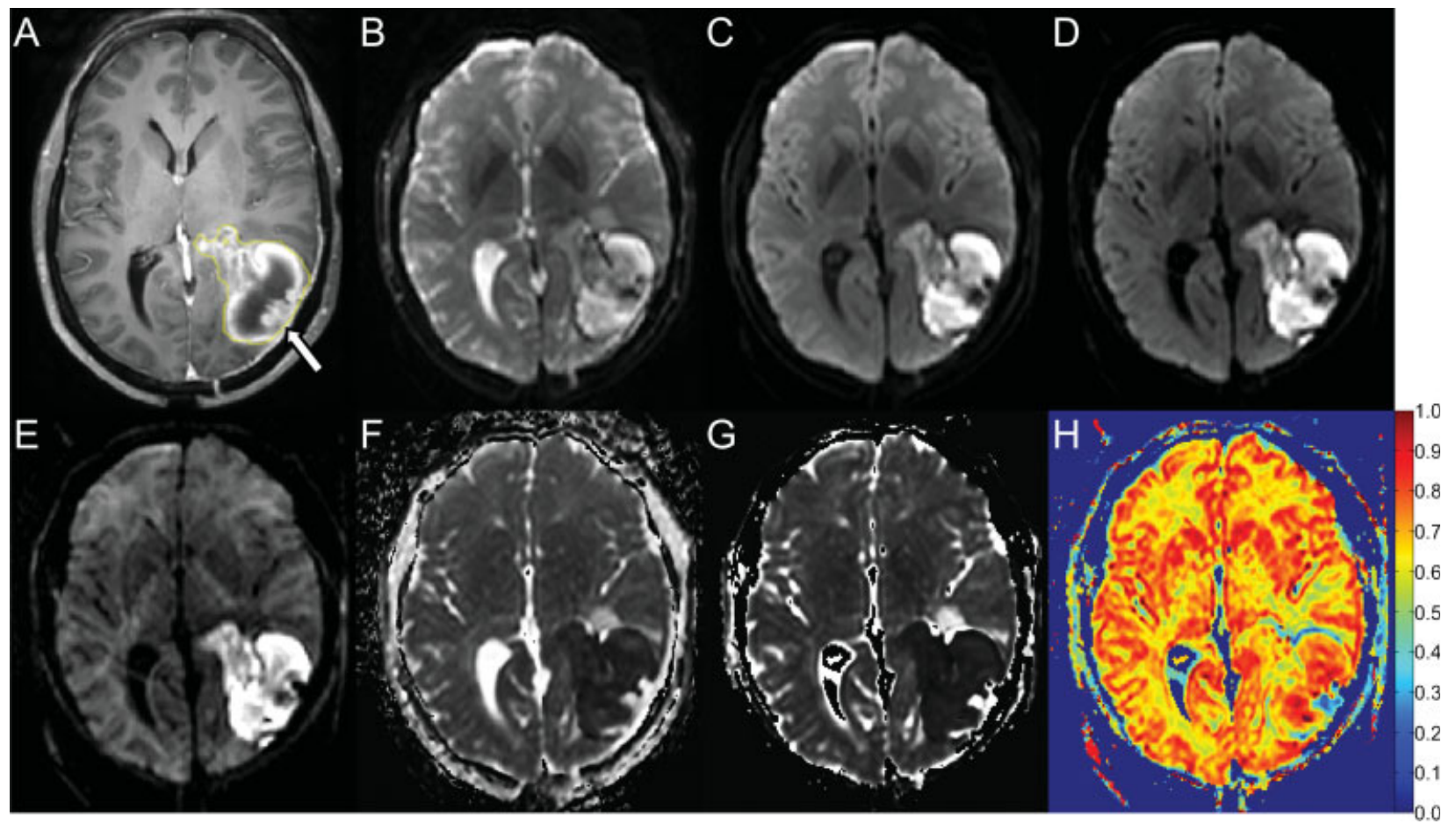

Figure 5. A 51-year-old female with a high-grade glioma (WHO grade IV) in the left occipital lobe. Axial post-gadolinium T1-weighted image with tumor VOI (a), diffusion-weighted images at b-values of $0 \mathrm{~s} / \mathrm{mm}^{2}$ (b), $1000 \mathrm{~s} / \mathrm{mm}^{2}$ (c), $2000 \mathrm{~s} / \mathrm{mm}^{2}$ (d), and $4000 \mathrm{~s} / \mathrm{mm}^{2}$ (e), and isotropic ADC (f), DDC (g), and $\alpha$ (h) maps show the tumor (arrow). This tumor had a mean DDC of $0.77 \times 0^{-3} \mathrm{~mm}^{2} / \mathrm{s}$ and a mean $\alpha$ value of 0.70 . In this case, overall contrast between the tumor and surrounding brain tissue was poor on the $\alpha$ map $(\mathrm{H})$; mean $\alpha$ values of the frontal white matter $(0.61)$ and of the white matter in the centrum semiovale (0.65) were even lower than that of the tumor (0.70). 

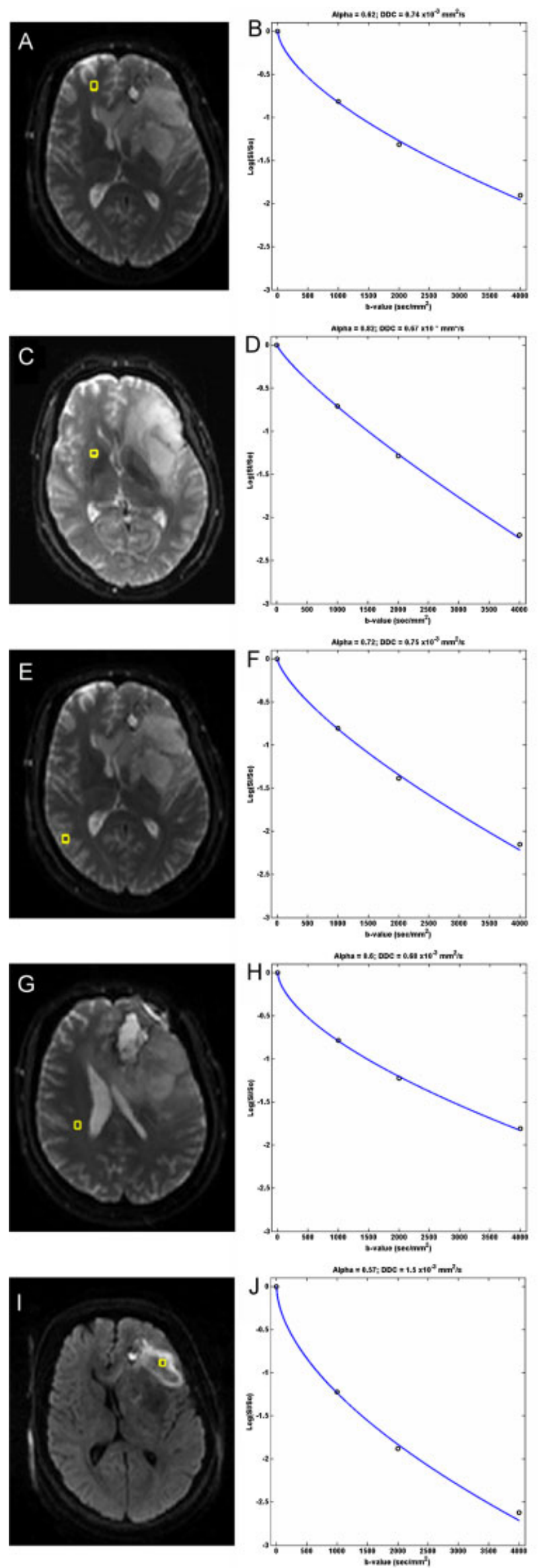

tumor $\alpha$ and tumor DDC were strongly negatively correlated (Fig. 1). It is well known that there is an inverse correlation between tumor cellularity and tumor diffusion coefficient (3). Combining these observations may suggest that $\alpha$ values of cystic or necrotic tumors are lower than those of highly cellular tumors. However, it should be noted that in the present study we selected more solid elements of the tumor by defining VOls based on contrast enhancement. Cystic cavities were only included in the tumor VOI if circumscribed by contrast enhancement and excluded if outside the enhancing region. In addition, high water mobility environments, such as cystic or necrotic components of tumor, typically did not retain sufficient SNR at $b=4000 \mathrm{~s} / \mathrm{mm}^{2}$ to be reliably fit by the stretched-exponential model. As described previously, a SNR-threshold filter was applied prior to model fitting to tag low SNR voxels as 'not-a-number' thus removing them from subsequent analysis. Thus, the observed negative correlation between the $\alpha$ value and DDC does not necessarily apply to the pure cystic/necrotic portions of the tumor. Furthermore, a drawback of the VOI-based averaging is the loss of macroscopic heterogeneity of local tissue in parameters ( $\alpha$ and DDC in this case). Therefore, despite our focus on more solid elements of the tumor, we would like to note there remains substantial macroscopic heterogeneity in tumor over individual VOIs as is well illustrated by the (wide range of) SDs of $\alpha$ and DDC values in Figure 1. Thus, further research is necessary to clarify the observed negative correlation between tumor $\alpha$ and tumor diffusion coefficient.

An important issue in the management of brain tumors is the selection of the most suitable biopsy target for accurate histopathological diagnosis (1-3). Since tumor grade is assigned on the basis of the region of highest grade anywhere in the tumor, an imaging test that could reliably depict such a site of highest tumor grade would be optimal for increasing the likelihood of correct diagnosis (1-3). Currently, selection of the biopsy target is usually based on the lesion's anatomic appearance and its contrast enhancement properties; biopsy is typically performed in an area of strong contrast enhancement. Interestingly, it is well-known that WHO grade is correlated to the degree of histological heterogeneity $(1,2,11)$. Since degree of histological heterogeneity may be well correlated to intravoxel diffusion heterogeneity, $\alpha$ maps may not only be useful for preoperative grading of brain tumors, but also for identifying the site of highest tumor grade for biopsy. This may increase the diagnostic yield of brain biopsies, which, in turn, may improve treatment planning and determining prognosis. On the other hand, the differences in $\alpha$ values between tumor and white matter was relatively small, and sensitivity of intravoxel diffusion heterogeneity for characterization of tumor grade may be of

Figure 6. Examples of the stretched-exponential model fit to target tissues in a 46-year-old male with a high-grade glioma (WHO grade IV) in the right frontal lobe (same patient as in Fig. 2). Axial isotropic $\alpha$ maps with regions of interest (ROIs) in the frontal white matter (a), basal ganglia (c), cortical grey matter (e), white matter in the centrum semiovale $(\mathrm{g})$, and tumor (i), and corresponding graphs with $\mathrm{b}$-value (x-axis) against logarithm of relative signal intensity (y-axis) for each $R O I(b, d, f, h, j)$. The mean $\alpha$ value of the tumor ROI (0.57) was lower than those of the ROls in the frontal white matter (0.74), basal ganglia (0.67), cortical grey matter (0.75), and white matter in the centrum semiovale (0.68). The more non-mono-exponential decay of the tumor relative to the other brain structures is well visualized by the degree of deviation from a straight line in the graph with b-value against logarithm of relative signal intensity $(H)$. 


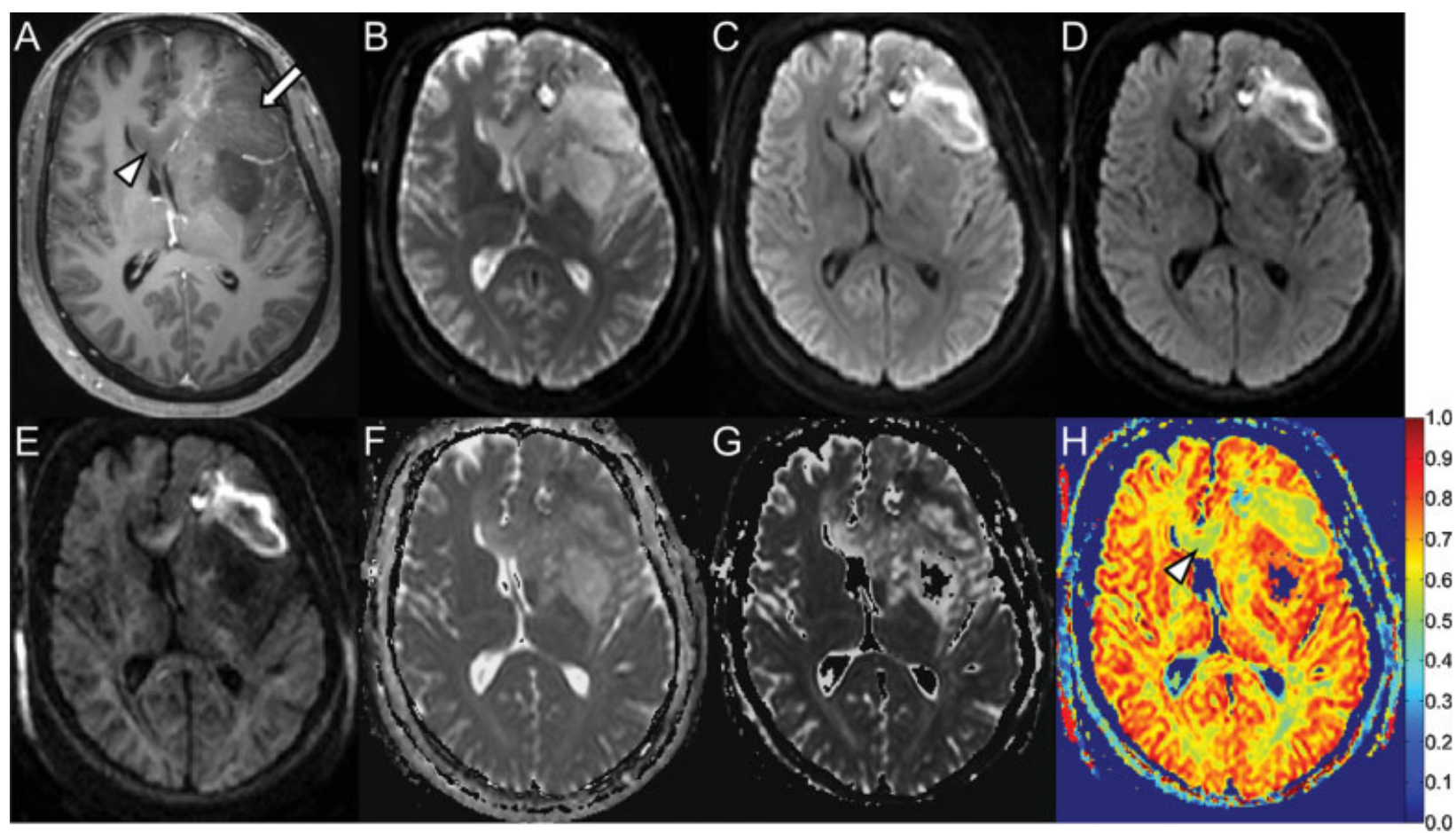

Figure 7. A 28-year-old male with a high-grade glioma (WHO grade III) in the left temporal lobe (same patient as in Fig. 2). Axial post-gadolinium T1-weighted image with tumor VOI (a), diffusion-weighted images at b-values of $0 \mathrm{~s} / \mathrm{mm}^{2}$ (b), $1000 \mathrm{~s} / \mathrm{mm}^{2}$ (c), $2000 \mathrm{~s} / \mathrm{mm}^{2}$ (d), and $4000 \mathrm{~s} / \mathrm{mm}^{2}$ (e), and isotropic ADC (f), DDC (g), and $\alpha(\mathrm{h})$ maps show the tumor (arrow). There is no evident contrast enhancement in the corpus callosum on the post-gadolinium image (arrowhead) (A). On the $\alpha$ map, however, a low $\alpha$ value can be seen in the corpus callosum (arrowhead) (H), suggesting tumor extension to the contralateral side of the brain.

concern (Figs. 4 and 5). Therefore, future animal/human studies with histopathological correlation are needed to confirm this theory.

Accurate local staging of the tumor is a requisite for appropriate locoregional therapy planning, including radiotherapy, chemotherapy, and surgery $(1,2)$. In particular, it is important to correctly exclude or confirm tumor involvement in the unenhancing region of peritumoral edema, but this is still an unsolved diagnostic problem (3). Furthermore, although highgrade gliomas rarely metastasize extracranially, it is well known that microscopic tumor spread at a considerable distance from the primary location may occur, which often remains undetected using conventional imaging (12,13). Bennett et al. (9) showed in an animal study that $\alpha$ values of peritumoral brain tissue were significantly lower than in the normal white matter. Fluorescence microscopy confirmed the presence of tumor cells in the peritumor region that could be histologically distinguished from the main tumor mass. Furthermore, there was no change on proton density or T2-weighted images in the peritumor region, making vasogenic edema, gliosis, or subacute peritumoral infarction unlikely as sources of contrast (9). However, in the present study, determining whether intravoxel diffusion heterogeneity imaging can diagnose tumor involvement in the unenhancing region of peritumoral edema would remain speculative because of the absence of histopathological correlation (3). Nevertheless, we incidentally observed findings that may support the use of intravoxel diffusion heterogeneity imaging in the assessment of tumor extent. For example, in one case the $\alpha$ index map suggested involvement of the corpus callosum and tumor extension to the contralateral side of the brain, despite the absence of contrast enhancement (Fig. 7). Other MR sequences supported this finding.

Currently, response to therapy in glioma patients is assessed using the MacDonald criteria, which are based on uni- or bidimensional changes in tumor size on enhanced MR imaging (14). Although volumetric tumor measurements have been reported to be more predictive of survival in recurrent glioma than uni- and bi-dimensional tumor measurements (15), tumor size measurements in general do not allow individualizing treatment, because the measurements are made well after the completion of therapy. Recently, a voxel-based quantitative DWI approach was introduced $\left(\mathrm{PRM}_{\mathrm{ADC}}\right)$, which allows visualization and calculation of tumor diffusion coefficient changes during treatment and takes into account spatial heterogeneity of tumor response (unlike mean diffusion coefficient measurements) $(4,5)$. $\mathrm{PRM}_{\mathrm{ADC}}$ combined with traditional radiological response criteria proved to provide a significantly better prediction of response to therapy than traditional radiological criteria or functional diffusion mapping alone $(4,5)$. Possibly, $\alpha$ of the tumor may also change during treatment (due to changes in histological heterogeneity). Further research is required to investigate this possible application.

Conventional imaging is often unreliable in distinguishing radiation necrosis from recurrent glioma in patients who are symptomatic after high-dose radiotherapy. ${ }^{18} \mathrm{~F}$-fluoro-2-deoxyglucose positron emission tomography (FDG-PET), MR spectroscopy, and perfusion imaging provide functional tissue information, and may be able to differentiate the two entities, but the most useful functional imaging modality has not been established yet $(3,16)$. Although the limited evidence indicates no substantial role for 
DWI (3), intravoxel diffusion heterogeneity imaging is based on other biophysical principles (image contrast is different from conventional DWI), and may be of value in distinguishing radiation necrosis from recurrent glioma. The potential of diffusion heterogeneity imaging in this field should be further explored.

MR imaging is the standard imaging modality for brain tumor evaluation, and DWI is one of the standard sequences in a brain tumor protocol (1-3). Therefore, practical implementation of intravoxel diffusion heterogeneity imaging is relatively easy, since it only requires adding a few more b-values $\left(>1000 \mathrm{~s} / \mathrm{mm}^{2}\right)$ to a conventional DWI sequence (which is usually performed using b-values of 0 and $1000 \mathrm{~s} / \mathrm{mm}^{2}$ ). Nevertheless, the present study is limited by the fact only four b-values were acquired, as it was designed to keep scan times reasonably short. Despite only four diffusion weightings, non-uniform b-value sampling was chosen to efficiently capture multi-exponential features at very high b-values along with the traditional b-values of 0 and $1000 \mathrm{~s} / \mathrm{mm}^{2}$. Furthermore, the imaging requirements for diffusion heterogeneity imaging also introduce some limitations and drawbacks. First, the use of SS-EPI (similar to conventional DWI) may lead to image distortion and signal loss (17). Although these artifacts have been considerably reduced thanks to the development of high-performance gradients and parallel imaging (18), they may still occur, especially at the base of the skull and the nasal sinuses, and evaluation of tumors in these areas using diffusion heterogeneity imaging may be hampered. In addition, the SNR characteristics of diffusion heterogeneity imaging are rather poor and voxel sizes tend to be large, which may hamper sensitivity to tumor cells occupying a small fraction of a voxel. Another disadvantage is that the use of high b-values $\left(>1000 \mathrm{~s} / \mathrm{mm}^{2}\right)$, necessary to render MR imaging sensitive to multi-exponential diffusion features, may impair the SNR on the $\alpha$ maps. The highest b-value $\left(4000 \mathrm{~s} / \mathrm{mm}^{2}\right)$ used in the present study was chosen based on available SNR and diffusion properties of the tissues of primary interest. High mobility environments, such as cerebrospinal fluid and necrotic cysts, did not maintain adequate SNR above background noise at high b-values $\left(\geq 2000 \mathrm{~s} / \mathrm{mm}^{2}\right)$ thus could not be reliably fit by the model. Low SNR voxels below a noise threshold were tagged as 'not-a-number' and eliminated from subsequent analysis (Fig. 2). Finally, fitting the stretched-exponential model to the DWI data to obtain whole-brain $\alpha$ map takes extra post-processing time, and is typically not included in clinical software packages.

A desirable feature of the stretched-exponential model is that it involves only two parameters (DDC and $\alpha$ value) in the fitting routine. The bi-exponential model has also been applied to fit to multi-exponential tissue data, but this model requires three parameters (two diffusion coefficients and the relative volume of each diffusion pool). Assuming for a moment both models are equally valid from a biophysical perspective, it is preferred to utilize a two-parameter model over a three-parameter model in fitting to so few data points. Other multi-exponential models have been developed in an attempt to characterize nonmono-exponential diffusion-weighted signal decay $(19,20)$. For example, Maier et al. (19) proposed quantifying the deviation of the signal attenuation from mono-exponential decay by means of the $\chi^{2}$ value, and showed that $\chi^{2}$ values of both peritumoral edema and highly malignant tumors were significantly higher than those of normal grey and white matter. Furthermore, Hall and Barrick (20) suggested describing diffusion-weighted signal decay in terms of the theory of anomalous diffusion, which assumes that the diffusion environment experienced by diffusing spins is not locally homogeneous. The $\gamma$ value derived from this anomalous diffusion model is comparable to the $\alpha$ value of the stretched-exponential model. In addition, MR diffusion kurtosis imaging was recently proposed to study the deviation of water diffusion from Gaussian distribution. An advantage of this method is that it only requires a modest increase in b-values beyond those typically employed for DWI (21). Nevertheless, although similarities exist among these models, acquired parameters of non-mono-exponential diffusion-weighted signal decay by each method should be interpreted differently, since theoretical biophysical underpinnings of each model are different.

A limitation of the present study is that $\alpha$ values of (apparently) unaffected contralateral anatomic landmarks were measured without the availability of histopathological correlation, and that these areas cannot definitely be considered free of tumor cells $(12,13)$. Furthermore, only high-grade gliomas were investigated, and it is still unclear whether there any significant differences between normal brain tissue and low-grade gliomas or other brain tumors. Despite these study limitations, the significantly different $\alpha$ values between anatomic landmarks and high-grade gliomas opens the path for several important potential clinical applications of diffusion heterogeneity imaging, as described previously.

In conclusion, the index $\alpha$ of human high-grade gliomas is significantly different from those of normal brain structures, which potentially offers a new method for evaluating brain tumors. The observed negative correlation between tumor $\alpha$ and tumor DDC requires further investigation.

\section{REFERENCES}

1. Behin A, Hoang-Xuan K, Carpentier AF, Delattre JY. Primary brain tumours in adults. Lancet. 2003; 361: 323-331.

2. Reardon DA, Rich JN, Friedman HS, Bigner DD. Recent advances in the treatment of malignant astrocytoma. J. Clin. Oncol. 2006; 24: 1253-1265.

3. Provenzale JM, Mukundan S, Barboriak DP. Diffusion-weighted and perfusion MR imaging for brain tumor characterization and assessment of treatment response. Radiol. 2006; 239: 632-649.

4. Hamstra DA, Galbán CJ, Meyer CR, Johnson TD, Sundgren PC, Tsien C, Lawrence TS, Junck L, Ross DJ, Rehemtulla A, Ross BD, Chenevert TL. Functional diffusion map as an early imaging biomarker for highgrade glioma: correlation with conventional radiologic response and overall survival. J. Clin. Oncol. 2008; 26: 3387-3394.

5. Hamstra DA, Rehemtulla A, Ross BD. Diffusion magnetic resonance imaging: a biomarker for treatment response in oncology. J. Clin. Oncol. 2007; 25: 4104-4109.

6. Maier SE, Bogner P, Bajzik G, Mamata H, Mamata Y, Repa I, Jolesz FA, Mulkern RV. Normal brain and brain tumor: multicomponent apparent diffusion coefficient line scan imaging. Radiol. 2001; 219: 842-849.

7. Bennett KM, Schmainda KM, Bennett RT, Rowe DB, Lu H, Hyde JS. Characterization of continuously distributed cortical water diffusion rates with a stretched-exponential model. Magn. Reson. Med. 2003; 50: 727-734.

8. Lee $\mathrm{JH}$, Springer CS Jr. Effects of equilibrium exchange on diffusionweighted NMR signals: the diffusigraphic 'shutter-speed'. Magn. Reson. Med. 2003; 49: 450-458.

9. Bennett KM, Hyde JS, Rand SD, Bennett R, Krouwer HG, Rebro KJ, Schmainda KM. Intravoxel distribution of DWI decay rates reveals C6 glioma invasion in rat brain. Magn. Reson. Med. 2004; 52: 994-1004.

10. Bennett KM, Hyde JS, Schmainda KM. Water diffusion heterogeneity index in the human brain is insensitive to the orientation of applied magnetic field gradients. Magn. Reson. Med. 2006; 56: 235-239. 
11. Kleihues P, Cavanee WK. World Health Organization Classification of Tumors. Pathology and Genetics. Tumours of the Nervous System. International Agency for Research on Cancer Press: Lyon, 2000.

12. Johnson PC, Hunt SJ, Drayer BP. Human cerebral gliomas: correlation of postmortem MR imaging and neuropathologic findings. Radiol. 1989; 170: 211-217.

13. Oh J, Henry RG, Pirzkall A, Lu Y, Li X, Catalaa I, Chang S, Dillon WP, Nelson SJ. Survival analysis in patients with glioblastoma multiforme: predictive value of choline-to- $\mathrm{N}$-acetylaspartate index, apparent diffusion coefficient, and relative cerebral blood volume. J. Magn. Reson. Imag. 2004; 19: 546-554.

14. Macdonald DR, Cascino TL, Schold SC Jr, Cairncross JG. Response criteria for phase II studies of supratentorial malignant glioma. J. Clin. Oncol. 1990; 8: 1277-1280.

15. Dempsey F, Condon BR, Hadley DM. Measurement of tumor 'size' in recurrent malignant glioma: 1D, 2D, or 3D? A.J.N.R. Am. J. Neuroradiol. 2005; 26: 770-776.
16. Palumbo B. Brain tumour recurrence: brain single-photon emission computerized tomography, PET and proton magnetic resonance spectroscopy. Nucl. Med. Commun. 2008; 29: 730-735.

17. Poustchi-Amin M, Mirowitz SA, Brown JJ, McKinstry RC, Li T. Principles and applications of echo-planar imaging: a review for the general radiologist. Radiograph. 2001; 21: 767-779.

18. Glockner JF, Hu HH, Stanley DW, Angelos L, King K. Parallel MR imaging: a user's guide. Radiograph. 2005; 25: 1279-1297.

19. Maier SE, Mamata H, Mulkern RV. Characterization of normal brain and brain tumor pathology by chisquares parameter maps of diffusionweighted image data. Eur. J. Radiol. 2003; 45: 199-207.

20. Hall MG, Barrick TR. From diffusion-weighted MRI to anomalous diffusion imaging. Magn. Reson. Med. 2008; 59: 447-455.

21. Jensen JL, Helpern JA, Ramani A, Lu H, Kaczynski K. Diffusion kurtosis imaging: the quantification of non-gaussian water diffusion by means of magnetic resonance imaging. Magn. Reson. Med. 2005; 53: 1432-1440. 